\title{
Introduction
}

\section{The Theoretical Context of This Study}

Dialogue tends to be employed as the principal means of communication, but I believe that without doubt there is a more cinematic manner of communicating.

-Stanley Kubrick ${ }^{1}$

The goal of this introductory chapter is to establish the theoretical context upon which this book is founded, starting with a discussion of a paradox that lies at the heart of what constitutes the central subject matter of this study, namely meaning in film. Second, we briefly discuss what has been the most influential model in film theory for dealing with this paradox, namely the linguistic model (also known as the film-as-language view). At the same time, we argue why this model is no longer sustainable in the light of the recent "embodied turn" in cognitive science. Third, we show how an embodied view of meaning forces us to address the paradox of cinematic meaning anew, thus prompting the need for a new research agenda. Fourth and last, we will lay out the main intentions and structure of this book as they emerge from the reorientation of the theoretical focus.

\section{The paradox of cinematic meaning}

This book is about meaning and cinema and the way this relationship is manifested in the films of the great American film director Stanley Kubrick (1928-1999). ${ }^{2}$ In this regard, Kubrick's oeuvre can be considered among the finest and most remarkable in film history. With films such as Paths of Glory (1957), 2001: A Space Odyssey (1968), Barry Lyndon (1975), The Shining (1980) and Eyes Wide Shut (1999), he created some of the most engaging cinematic artworks in modern cinema that continue to fascinate audiences and critics today. ${ }^{3}$ In attempting to explain this endless fascination with Kubrick's work, scholars have recurrently pointed toward the filmmaker's ability to shape its conceptual content in an almost exclusively visual way. 2001: A Space Odyssey is perhaps the purest embodiment of this thought. Out of two hours and nineteen minutes of film, there are only a little less than forty minutes of dialogue, yet, the film conveys a richness and complexity of themes rarely equalled in other films. As Michael Benson recently stressed in his book, exactly fifty years after its release: " 2001 is essentially a nonverbal 
experience, once more comparable to a musical composition than to the usual dialogue-based commercial cinema. ... It spoke its own language, ... the authority and power of the images themselves didn't necessitate literal comprehension." ${ }^{\prime}$ This refusal to fit meaning into the "straitjacket of words," as Kubrick calls it, also runs as a red thread throughout the interviews that were conducted with the director over the years. ${ }^{5}$ Cited below is one excerpt from Kubrick's comments, as it appeared in 1969 in the magazine Action:

\begin{abstract}
In Space Odyssey the mood hitting you is the visual imagery. The people who didn't respond, I now, for want of coming up with a better explanation, categorize as "verbally oriented people." ... Communicating visually and through music gets past the verbal pigeonhole concepts that people are stuck with. You know, words have a highly subjective and very limited meaning, and they immediately limit the possible emotional and subconscious designating effect of a work of art. Movies have tied themselves into that because the crucial things that generally come out of a film are still word-delivered. There's emotion backing them up, you've got the actors generating feeling, etc. It's basically word communication. ${ }^{6}$
\end{abstract}

However intuitively true the attribution of themes or meanings to the non-verbal, perceptual level of Kubrick's cinema may sound, the less clear it is from a purely logical and theoretical point-of-view. That this attribution is less evident than it appears at first sight becomes clear once we isolate the premises on which it is founded:

(1) Films present the opportunity to communicate abstract meanings without the traditional reliance on words. ${ }^{7}$

(2) Meaning is a matter of conceptual structure.

(3) Films, as opposed to words, do not connect so easily to concepts. ${ }^{8}$

(4) How, then, can films be capable of communicating conceptual meaning?

So despite the fact that premise (1) sounds intuitively true, it bears a set of premises, (2) and (3), that, apparently, seem to contradict each other. We shall label this logical inconsistency, which leads again to a questioning of the relationship between meaning and cinema (4), the paradox of cinematic meaning.

Let us start our investigation of this paradox by considering the question underlying the first premise: On what conditions does successful communication of meaning depend? Perhaps the most straightforward answer to this question has been provided by the British philosopher Paul Grice. In his influential article from 1957 called "Meaning" the author has argued that communication of meaning is successful insofar the perceiver of the representation (e.g., the hearer) understands the representation that is being communicated (e.g., the utterance), that is, and here is where the central claim of his argument becomes manifest, insofar the perceiver recognizes the communicator's intention to represent, and further recognizes that he himself is intended to recognize it. In Grice's own words, "for A to mean something by $\mathrm{x}, \mathrm{A}$ must intend to induce by $\mathrm{x}$ a belief in an audience, and he must also intend his utterance to be recognized as so intended." This aspect is also known as the "self-referentiality" of the intention to communicate and is, as the American philosopher John Searle pointed out fifty years later, "seldom remarked on." ${ }^{10}$ The crucial question, then, is this: if successful communication of meaning depends on the audience's recognition of the communicator's intention to represent the meaning, how then can this recognition be achieved? The key to answering this question lies in the representation $\mathrm{x}$. 
Here we may quote Noël Carroll, who adds the following note to Grice's analysis: "The intention A intends to be recognized must be discernible in $\mathrm{x}$. Where $\mathrm{x}$ is an artwork, the intention the artist means to convey must be discernible in the work." ${ }^{11}$ If we further define this intention in terms of mental conceptual structure (let us call this $\mathrm{y}$ ), it follows that $\mathrm{y}$ has to be imposed onto $\mathrm{x}$ for it is only when $\mathrm{y}$ is embodied in $\mathrm{x}$ that the audience will be able to extract $y$ from $x$, and thus achieve recognition of the communicator's intention. ${ }^{12}$

The conception of meaning and communication just sketched out is not a new one, but it echoes the underlying theoretical assumptions of two different, but neighboring areas of research, namely cognitive semantics and inferential pragmatics. The first discipline began in the 1970s and initiated a radical critique of the truth-conditional view of meaning in language, as advocated by the Anglo-American tradition in philosophy. ${ }^{13}$ This view rests upon the assumption that meaning can be objectively described as a relationship between words and an objective external reality, and that this relationship can be modelled in terms of truth or falsity. ${ }^{14}$ Cognitive semantics, as put forth by such scholars as Leonard Talmy, George Lakoff, Mark Johnson and Ronald Langacker, rejects this view, which inevitably leads to an undervaluation of the role of the mind, and asserts instead that semantic structure (i.e., the meanings conventionally associated with words) can be equated with conceptual structure, "the nature and organisation of mental representations in all its richness and diversity." ${ }^{15}$ Moreover, cognitive semantics claims that this conceptual structure is fundamentally embodied. This principle is known as the thesis of the "embodied mind" and roughly states that the nature of conceptual content emerges from bodily experiences and interactions with the environment. ${ }^{16}$

The second discipline began to flourish in the late 1970s and 1980s and initiated an alternative to the classical code model of communication, according to which utterances are signals that encode messages and comprehension is achieved by decoding the signals to obtain the messages. On the inferential view, originally suggested by Grice, but further developed by such scholars as Wilson and Sperber, representations such as utterances are not signals, but pieces of evidence about the speaker's meaning, and comprehension is achieved by inferring this meaning from evidence provided by the representation and the context in which it is produced. ${ }^{17}$

What quality, then, does the representation need to possess in order for it to express and externalize the conceptual structure? The general answer is that the representation has to "connect to" the conceptual structure. As for language, the key focus of both cognitive semantics and inferential pragmatics, this connection is inherent to its symbolic function. When we use language and write the word "tree," the meaning conventionally paired with it, is not the particular physical object of a tree, but the idea of a tree, that is, the concept of a tree. ${ }^{18}$ As a result of this pairing of form and concept, language is often taken at face value when discussing the process of transmitting meaning from one entity to another. This is evidenced in the many references people make to language when talking about the phenomenon of communication itself (i.e., our meta-language). Consider, for example, the following list of English expressions, as compiled by the cognitive linguist Michael Reddy:

Whenever you have a good idea practice capturing it in words. You have to put each concept into words very carefully. Try to pack more thoughts into fewer words. Insert those ideas elsewhere in the paragraph. Don't force your meanings into the wrong words. ${ }^{19}$

As Reddy argued, these expressions can be seen as linguistic manifestations of a general metaphor system which he coins the "conduit metaphor." According to this metaphor, people, when communicating, "insert" internal 
xviii | Introduction

concepts (e.g., ideas, thoughts, emotions) "into" external "containers" (e.g., words, phrases, sentences, etc.) whose contents are then "extracted" by listeners and readers. Because language allows for a symbolic assembly of form and meaning, it is only natural to refer to words and paragraphs as the proper "insides" wherein the meanings can reside. Diagrammatically, this "trajectory" from mind to language might be represented as in figure I.1 by means of an arrow running from one container to another. The first part of the trajectory designates an EXIT path: the conceptual meaning goes from inside the communicator's head (the body as container for the mind) to its outside. The second pattern, by contrast, describes an ENTRY path: the meaning goes from outside the communicator's head to the inside of language. ${ }^{20}$ As stated, this ENTRY path is facilitated by the symbolic function of language.

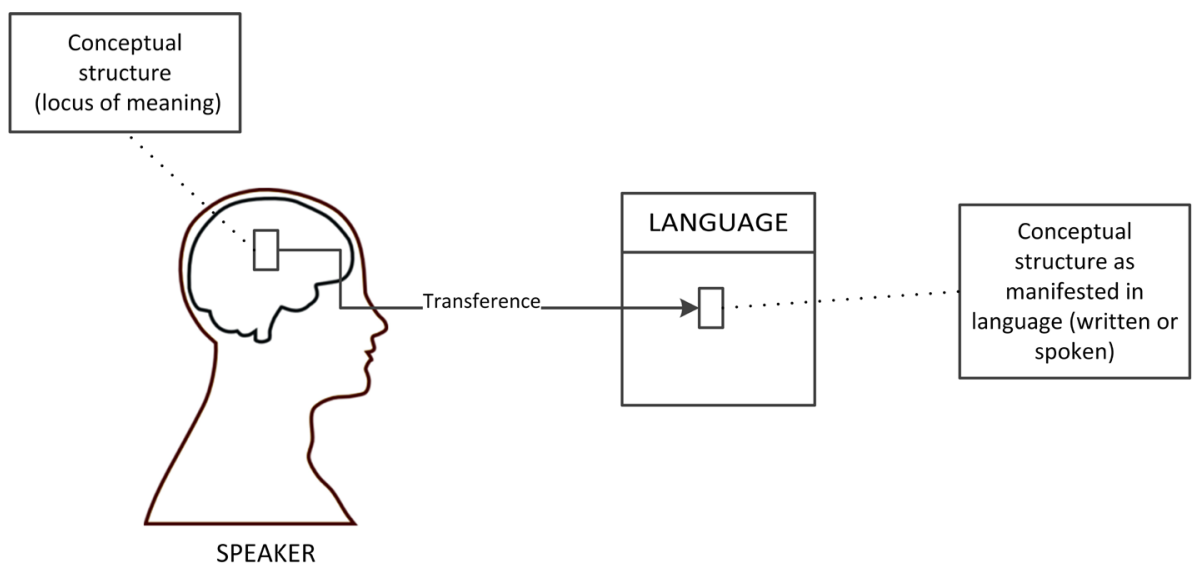

Figure I.1 Language as the "conduit" of conceptual structure.

A look at film, however, reveals a far more complicated picture. First, there is the question of identifying the communicator. Who is the agent who intentionally makes an utterance in a medium that usually implies the contribution of more than one individual? Raising this question brings us to the much-complicated matter of authorship in cinema. ${ }^{21}$ As Sellors aptly points out, "authorship is a problem in film studies that simply will not go away." 22 Exploring this debate lies beyond the scope of this book. On a general note, it is sufficient to say that whoever the communicator in film may be, whether it be an individual mind or a collective of minds, it does not change anything to the central principle of cognitive semantics that meaning is equated with conceptual structure. In other words, it is less important to know to which "concrete" individual artist (e.g., filmmaker) the conceptual structure can be attributed than to assume for now that it is the conceptual structure that is being manifested in the representation, whether it be an utterance or, as in our case, a film. Following Turner's book, we may call this general and unspecified mind to which the conceptual structure adheres "the artful mind."23

Having said this, Kubrick, however, presents us with a rather unique case in motion-picture history if it comes to defining authorship in cinema. As has been repeatedly stressed in the literature, Kubrick, more than any other major filmmaker working within the context of a studio system, was able to maintain an uncommon high degree 
of independence and directorial control in the sense of decision-making authority and responsibility with regard to the making and overall design of his films. As Philips writes: "By steadily building a reputation as a filmmaker of international importance, he gained full artistic control over his films, guiding the production of each of them from the earliest stages of planning and scripting through post-production." ${ }^{24}$ Because Kubrick stood much closer to his material than almost any other filmmaker working in Hollywood, it is not surprising, as Young already observed in 1959, that there is a "strong feeling of unity and single-mindedness in his films." ${ }^{25}$ Although such a result can never be guaranteed given the collective nature of film-making, Kubrick's unique reputation allows us, in other words, to speak of Kubrick as a "cinematic author" or a "filmic author," in the senses defined by Livingston and Sellors, respectively. ${ }^{26}$

The critical reader, however, might object here that we are putting too much emphasis on the filmmaker's or artist's or speaker's intention. Indeed, does the meaning available in films often not exceed the artist's intention? Do we not value the work partly because it enacts possibilities of meaning that go beyond anything that the speaker or filmmaker consciously intends? This is a very good point, and therefore, we have to be very clear from the start about the sort of meaning that this book will be engaged in. To sort this out, we may turn to Bordwell's distinction between "referential and explicit meanings," and "implicit and symptomatic meanings." ${ }^{27}$ The former constitute the backbone of narrative comprehension as they fall together with the "apparent, manifest, or direct meanings" of a work. ${ }^{28}$ They are close to the bare-plot summaries of the films as they largely result from the viewers' attempts to construct a mental model of the situation in which the narrative action takes place. Van Dyck and Kintsch call this the "situation model." ${ }^{29}$ Spectators construe such models by drawing not only on their knowledge about conventions, but also and more profoundly, as chapter 1 will make clear to us, on conceptions of causality, space and time. As Persson points out, it is an important feature of the situation or the referential meaning that it is closely tied to the "spectators' abilities to understand the behavior in terms of character psychology" and to infer "causal relations between events and scenes," which often involve "a character's mental states and traits" (e.g., "Alice is angry with her husband Bill because he did not get jealous when she told him that another man wanted sex with her," "Alex feels sick when he watches violence on the screen," "Wendy is shocked when she sees the word REDRUM in the mirror," "HAL 9000 decides to terminate the astronauts Bowman and Poole because he thinks they want to disconnect him"). ${ }^{30}$ In the field of philosophy of mind these causal relations involving mental events are known as instances of "mental causation." ${ }^{11}$ This concept provides us with a thick and rich level of meaning that is central to our understanding of narratives, including, as we shall see in chapter 1, the narratives of Kubrick's work.

The latter, by contrast, are more "hidden" and "non-obvious," and have to do with the process of interpretation. ${ }^{32}$ At this level, we enter a more abstract and symbolic understanding of cinematic meaning. They often contain speculations and claims about "how the film supposedly is bound up with certain ideas, values, or ideologies than in itself is not 'aware of"' (e.g., "The monolith is Kubrick's representation of the cinema screen itself," "The Shining is about the genocide of Native Americans," "Eyes Wide Shut is rife with Illuminati symbolism"). ${ }^{33}$ Although these symptomatic meanings or meanings "against the grain" emanate out of the film, many of them operate outside the film's diegetic and fictional world. They "take a step back," as Persson writes, "from the film, investigating its fictional, narrative, communicatory, rhetorical, and societal functions rather than establishing its fictional meaning." ${ }^{44}$ As Bordwell and Thompson have stressed, the abstract qualities of such implicit meanings "can lead to very broad concepts often called themes." ${ }^{35}$ Many of Kubrick's films seem to exhibit the theme of dehumanization. How valuable such descriptions may be, they nevertheless stay very general; they fit for literally 
hundreds of films. Therefore, Bordwell and Thompson suggest that "the search for implicit meanings should not leave behind the particular and concrete features of a film... . we should strive to make our interpretations precise by seeing how each film's thematic meanings are suggested by the film's total system." ${ }^{36}$ A good scholarly example of such a combined incorporation of thematic interpretation and close formal analysis, can be found, for instance, in Robert Kolker's seminal chapter on Kubrick entitled "Tectonics of the Mechanical Man." ${ }^{37}$ The work explicitly adopts an ideological and cultural approach, yet the thematic wanderings offered by the author never lose touch with the formal evidence offered by the filmmaker's work. ${ }^{38}$

Although the line between comprehension and interpretation is not always easy to draw, it will be the referential or situational meanings that will be the primary focus of this study for the basic reason that these are concerned with the "overt facts about story or theme that are directly presented as such within the film." ${ }^{39}$ In other words, if we wish to show how films are capable of conveying meaning non-verbally, then it is best to focus on the sort of meaning of which we are certain to a confident degree that it is actually intended to be communicated by the films to the viewer. Situational meanings and plot summarization largely meet this condition and are therefore most appropriately fitted to examine the question of meaning in film. Speculations about the philosophical and allegorical meanings of the film, on the other hand, how interesting as they might seem, do not always offer this degree of specificity and are, in this sense, less appropriate. Not surprisingly, the "lowest," literal level of meaning, that of straightforward explanation of the plot, was also the only level of meaning that Kubrick was keen to discuss himself as he rightly felt that a verbal summary of the "deeper" meaning was not only impossible, but also deceptive given the fact that it is intended to involve the audience in an experience. As he once stated in an early interview: "Films deal with the emotions and reflect the fragmentation of experience. It is thus misleading to try to sum up the [deeper] meaning of a film verbally." 40

By focusing upon intended situational meanings, do we not disregard the emotional and subconscious effects that Kubrick's cinema seem to emphasize? This is correct only if one assumes that the one has nothing to do with the other. As soon will become clear, however, such a distinction is not something that this book intends to maintain. Indeed, it will be one of the key objectives of this book to demonstrate that much of the intended visual situational meanings in Kubrick's film have their roots in bodily and nonconscious meanings that escape any intentional verbal articulation. In other words, the focus upon situational meanings should be seen as a way (and an opportune way at that) to reveal the cognitive unconscious dimensions of meaning-making that so forcefully account for our endless fascination with Kubrick's films.

But we must not run ahead of our argument. Now that we have sorted out the kind of meaning that this book will take as a starting point, let us further stress two more basic observations that may account for a film's complexity. The first one is that film, as opposed to language, can be conceived as a container for many other subcontainers: one for each mode of representation it contains (a visual container, a gestural container, a musical container, a linguistic container, etc.). In other words, the "trajectory" of meaning that runs from the conceptual and mental level to the external level of representation does not develop in one direction as it is the case with language, but in various directions, thus giving rise to many potential ENTRY paths. Moreover, these paths do not co-exist as parallel lines. Belonging to the generic container called "film," they are interconnected thus influencing each other in various ways.

The second observation is that many of these subcontainers have a profoundly different ontological status than language. Take, for instance, the visual subcontainer, the one most relevant to our understanding of cinema. It has 
been frequently noted in the literature that pictorial representations, as opposed to words, maintain a relationship with the represented reality that is based on resemblance rather than on arbitrary convention. ${ }^{41}$ They are what semioticians call iconic signs instead of symbolic ones. ${ }^{42}$ Although iconic signs do not literally possess the properties of the represented or denoted object, they nevertheless seem to "reproduce" some of its properties. ${ }^{43}$ In film studies this is often further explicated in causal terms. As Gaut writes, "we speak of a photograph of some object only if that object caused a light pattern to be imprinted on the photographic emulsion." ${ }^{4}$ This causal relation, the author points out, is not arbitrary, but "fixed by empirical facts." In other words, if the symbolic function of language facilitates the transference of concepts, and this function is absent from iconic images, how then can these images connect to conceptual structure?

The picture becomes even more complicated when we consider the subcontainer of music ("pure" or "absolute" instrumental music, that is), which appears to be quite different from the standard representational arts, such as (figurative) painting, photography and literature. As Scruton asks himself, "is there anything, other than itself, that music means?" 45 For this reason, because music lacks a clear object or reference, music has often been characterized as "abstract." ${ }^{46}$ Consequently, if the representational capability of music is questioned and this capability is conditional for communicating meaning, how then can music become a container for meaning? It is a question frequently posed, but seldomly answered in a manner that is satisfying.

It should be obvious by now, then, that the "entrance" question of meaning is much more complicated in film than it is in language. It is at this point in our argument that we can see how the paradox of cinematic meaning starts to emerge: Film seems to lack the form-concept pairing that makes symbolic language such a suitable container for the storage of meaning, yet scholars and layman alike assume that film, just like language, is capable of conveying meaning. In a diagrammatical way, this may be visualized as in figure I.2.

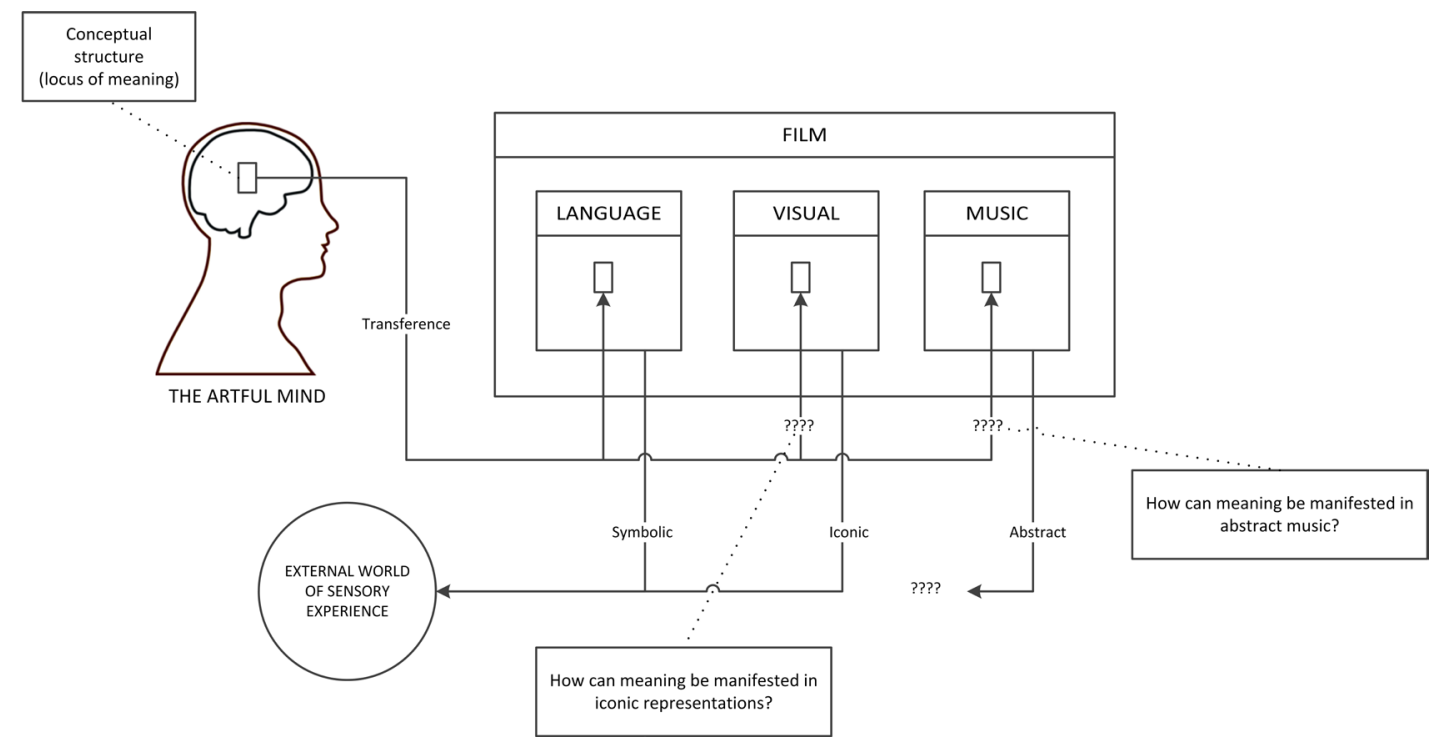

Figure I.2 The paradox of cinematic meaning. 
Given this diagram, then, one may argue that the key challenge lies in finding a way to reconcile the conceptual structure of meaning with the distinctive representational structure of film by facing such questions as: How can conceptual structure be bridged to the iconic surface of visual representations? How can music be meaningful while at the same time being non-representational? How do the answers to these questions interact with each other within the generic container called film? Yet, from early on, film scholars have predominantly preferred to evade these questions by pursuing another challenge, one that is not so much motivated by the essential differences between film and language, but by the question of how meaning in film can be modelled upon linguistic, symbolic meaning. The broad metaphor used to describe this linguistic turn in film studies has come to known as the FILM AS LANGUAGE metaphor.

\section{The film as language metaphor}

Ever since the birth of cinema it has been customary to talk about film as if it were a "readable text" with its own "syntax" and "grammar." References to linguistic terminology can be traced back to the earliest writings on film, to reach its height in the 1970s with the rise of film semiotics. ${ }^{47}$ Taken together, phrases such as "the cinematic text," "cinema speech," "the grammar of film" or "the language of film" provide linguistic evidence for the existence of the FILM AS LANGUAGE metaphor. ${ }^{48}$ This metaphor presumes a set of cross-domain mappings between the source domain of language and the target domain of film (usually restricted to the visual subcontainer), some of which are summarized as in table I.1.49

Table I.1 The FILM AS LANGUAGE metaphor.

\begin{tabular}{ll}
\hline Source domain [Language] & Target Domain [Film] \\
\hline Text & Film \\
Reading & Film comprehension \\
Words & Shots \\
Sentences & A montage sequence \\
Syntax & Principles for combining shots \\
\hline
\end{tabular}

It is not difficult to see why this metaphor is so appealing. Because most of us assume that film can be meaningful in the same way that language is meaningful, it makes intuitive sense to draw on linguistic terminology to describe our understanding of film. But what does this analogy substantially mean? What does it mean to say that a non-symbolic medium such as film can be compared to a symbolic medium such as language and more importantly what are its consequences for the conception of meaning in cinema? To avoid any misunderstanding, it might be useful to first distinguish, as John Carroll did, between two general ways of interpreting the analogy between film and language, namely as a strong theoretical claim or as a weaker methodological assumption. ${ }^{50}$ The theoretical claim asserts that language and cinema resemble one another directly. They are conceived of as 
members of the same natural kind. The methodological assumption, by contrast, asserts no such theoretical claim, but instead emphasizes the methodological value of using linguistic models as a means for guiding film theory. The theoretical claim seems hard to defend for a number of obvious reasons which we will not elaborate on here. Therefore we will only limit ourselves to the justification of the methodological argument. To see its appeal, let us consider, for example, the following series of images, as cited from the introduction to Carroll's book:

(1) A close-up shot (i.e., face only) of a man, A, smiling.

(2) A medium-shot (i.e., from the waist up) of two men, A and B, engaged in conversation.

(3) A long-shot (i.e., revealing both men completely) of the two men A and B parting; they wave to one another as they walk off. ${ }^{51}$

The order above implies that the smiling gesture of A in (1) invited the conversation. However, as Carroll further points out, if we should put the same images in a different order, we would get an entirely different meaning: the order (2), (1), (3) suggests that something in the conversation pleased A, whereas the order (2), (3) and (1) suggests A's overall satisfaction with meeting $B$. This observation led many scholars to believe that the conveyance of meaning in film works quite similar to the conveyance of meaning in language, that is, just like the meaning of a sentence depends on the order of the individual words, so does the meaning of a scene depend on the order of the individual images. Since the discipline of structural linguistics, as it was founded by Ferdinand de Saussure, was considered to be the most sophisticated discipline for analysing a discourse's underlying reality, it was only logical, from a methodological point-of view, that its conceptual tools should also be extended into the realm of film. Hence, the birth of classical film semiotics. ${ }^{52}$ Of major importance in its development were the writings of Christian Metz who, in the 1960s and 1970s, undertook the task of lending the methodological assumption of the FILM IS LANGUAGE metaphor more theoretical weight by modelling film's underlying reality on the rules and structures governing the linguistic sign. As Metz himself put it, "the task of the semiotics of the filmic fact" is "to analyse film texts in order to discover either textual systems, cinematic codes, or sub-codes." ${ }^{53}$ It would take us too far afield to consider the methodological issues of such an attempt in detail. For our present purpose, however, it is more important to consider the theoretical implications of such an undertaking for the concept of meaning in cinema. In the previous section, we already assumed that meaning is fundamentally mind-dependent, that is, in order for a representation to be meaningful, the representation has to connect to the internal conceptual structure of the mind (i.e., the representation as the manifestation of conceptual structure). Comprehension occurs when the observer is able to infer this content on the basis of the evidence provided by the representation. This requires a degree of recognition which can only happen if the representation externalizes the conceptual structure. This, in turn, led us to formulate the ontological paradox of cinematic meaning: how can film externalize concepts given that film, for its largest part, does not connect to concepts as language does through its symbolic form? By contrast, the linguistic approach to meaning, such as the one initiated by Metz, is fundamentally mind-independent. Its dependence on the linguistic notion of a sign adheres primarily to a relational and objective conception of meaning according to which meaning is based on differences between signs. 
But how, then, can film semiotics provide us with a satisfying account of meaning in cinema, if we assume that meaning is unavoidably tied to the conceptual structure of the mind? The answer here is as follows: a linguistic approach to meaning can only be justified insofar it is supported by a science of the mind that similarly puts the arbitrariness of the sign at the centre of its theoretical claims. Such an objectivist approach to psychology was provided in the 1950s with what now is commonly referred to as "first-generation cognitive science" or "the cognitive science of the disembodied mind." ${ }^{54}$ Having its roots in artificial intelligence, information-processing psychology, analytic philosophy of mind and language, and Noam Chomsky's idea of an innate grammar, it assumed a view according to which the mind is symbolic and algorithmic. ${ }^{55}$ As Johnson writes: "Mind was taken to be a capacity for formal operations and functions that was not dependent on any one particular form of embodiment." ${ }^{36}$ Because these formal symbols of the mind bear a relationship with perceptual experience that is arbitrary, the comparison to language was easily made. This idea lies at the heart of Fodor's hypothesis of a "language of thought," which presumes an infinite set of mental representations that "acquire their meaning by being 'about' —or referring to-the states of affairs in the external world." ${ }^{77}$ Similarly to how words typically have arbitrary relations to entities in the world, these symbols of the mind have arbitrary relations to perceptual states. It is precisely for this reason that Barsalou calls these symbols "amodal." "Just as the word 'chair' has no systematic similarity to physical chairs, the amodal symbol for chair has no systematic similarity to perceived chairs." 58 As Johnson points out, this (false) idea that all human thinking has the form of a language is deeply entrenched in our ordinary and philosophical discourse. ${ }^{59}$ Because it is so common for humans to express their thoughts in language (recall Reddy's "conduit metaphor"), "we are easily seduced into believing that the operations of mind and thought are structured like the operations of written and spoken language." ${ }^{00}$ We presuppose, as Lakoff and Johnson label it, the THOUGHT AS LANGUAGE metaphor (see table I.2). ${ }^{61}$ This metaphor is evidenced in expressions such as "Let me make a mental note of that," "She's an open book to me-I can read her every thought," "The public misread the President's intentions," and "Do you think I'm some kind of mind-reader?"

Table I.2 The THOUgHT AS LANGUAGE metaphor (after Lakoff and Johnson).

\begin{tabular}{ll}
\hline Source domain [Linguistic Acts] & Target Domain [Thinking] \\
\hline Linguistic activity (speaking/writing) & Thinking \\
Words & Ideas \\
Sentences & Complex ideas \\
Spelling & Communicating a sequence of thoughts \\
Writing & Memorization \\
\hline
\end{tabular}

Hence, if the mind is believed to share a formal language consisting entirely of arbitrary symbols, and meaning is conceived to be a matter of the mind, it follows that all meaning is linguistic meaning, including meaning in film. As to the definition of linguistic meaning, approaches differ. As we already saw, Saussure viewed linguistic meaning as essentially based on differences within language. By contrast, truth-based approaches to linguistic meaning conceive meaning as a relation between words and objective (mind-independent) reality. ${ }^{62}$ According 
to this "objectivist theory of meaning" conventional and arbitrary signs such as words become meaningful insofar they refer to the state of affairs in the world (e.g., things, persons, events). Despite these differences, they nevertheless share one core assumption, namely that meaning is best captured in terms of a conception of human thought that, similar to the arbitrary nature of language, is disembodied, that is, a mind separated from its body and its world. ${ }^{63}$

Consequently, by providing a conception of the mind within the language analysis tradition, first-generation cognitive science provided film semioticians with "the ideal paradigm" for their linguistic method. ${ }^{64}$ This can be derived from the following line of reasoning:

(1) Meaning is a matter of conceptual structure.

(2) Like language, this conceptual structure is arbitrary and disembodied (i.e., the THOUGHT AS LANGUAGE metaphor).

(3) Hence, meaning can be equated with linguistic meaning.

(4) If film wants to have true meaning it has to be modelled on linguistic meaning.

(5) Hence, in order to study this meaning, one has to consider the semantic or syntactic rules that govern the non-perceptible system underlying film (i.e., the research aim of film semiotics).

As Buckland has pointed out, the engagement of film semiotics with first-generation cognitive science, in particular Noam Chomsky's transformational generative grammar, has led a number of European film theorists, among them Chateau and Colin, to overcome what he coins the "translinguistics of Metz's film semiotics," that is, "Metz's insistence that film semiotics be based exclusively on the methods of structural linguistics." ${ }^{\prime 5}$ Buckland refers to this next "maturation" stage of semiotic film theory as the "cognitive semiotics of film."

More importantly, however, from the perspective of this book, first-generation cognitive science and the mind-body dualism that underpins it, provided film semioticians with a scientific and formal argument for overcoming the paradox of cinematic meaning. Since Fodor's language of thought metaphor seduces us to believe that conceptual structure has the form of a language (i.e., not a natural one, but a formal one), it is no longer necessary to consider the mind as the locus of meaning. Rather, it is the linguistic sign that now takes on this role as it becomes the epitaph by which film connects to language. In this sense it can be said that film semiotics provides a solution to the paradox of cinematic meaning. Film is meaningful because its underlying reality is governed by the same disembodied and media-independent rules of meaning-making that are manifest in verbal language (and in all other media for that matter). Schematically, this can be represented as in figure I.3. 


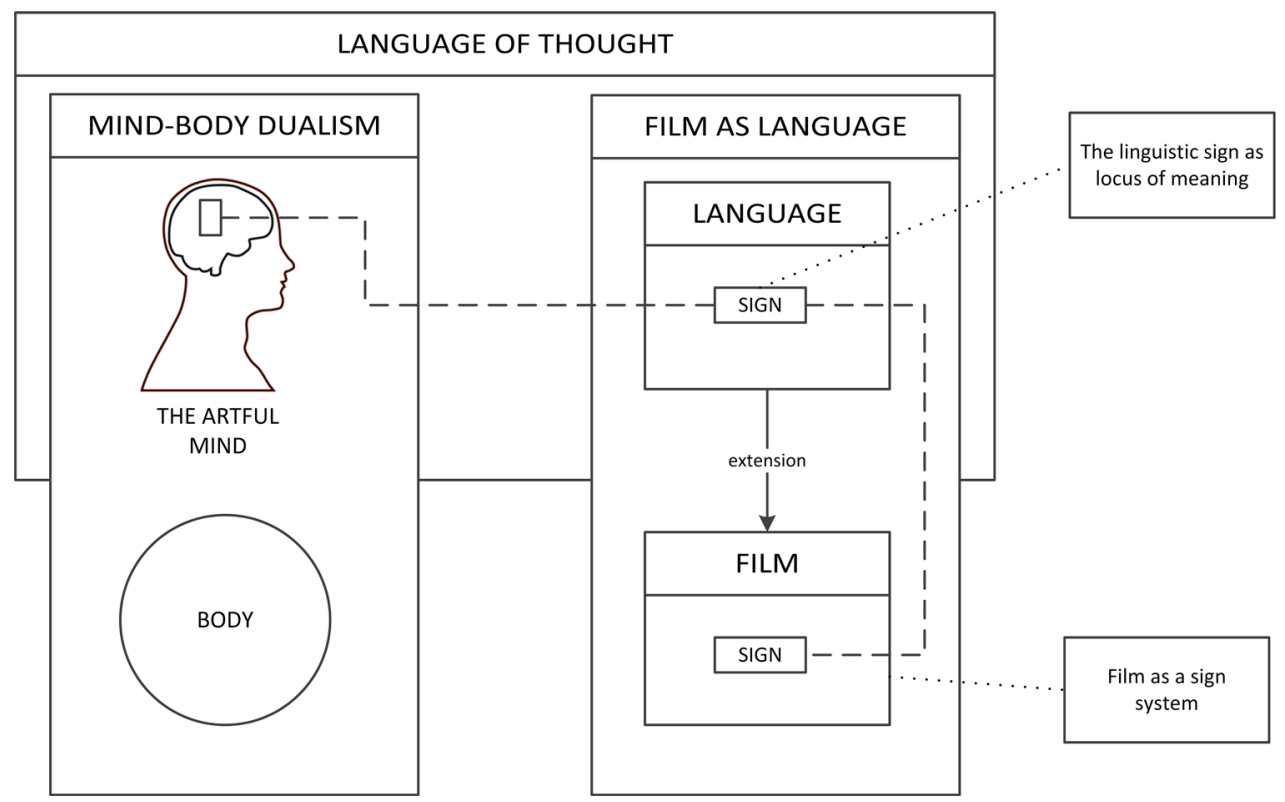

Figure I.3 Evading the paradox of cinematic meaning: The FILM AS LANGUAGE metaphor.

Over the last two decades the linguistic approach to meaning in film has met with increasing criticism, especially from Anglo-Saxon quarters. ${ }^{66}$ Part of it has been directed to the strong theoretical claim of a direct resemblance between film and natural language. ${ }^{67}$ This point of criticism, however, seems rather ill placed, as Buckland has counter-argued, because Metz himself has repeatedly stated that film is not analogous to natural language ("film is a language sans langue"). ${ }^{68}$ As we have seen, film semiotics was not so much founded on any direct resemblance between film and language, but on methodological grounds, the idea that film's specific, underlying reality could be reconstructed by the methods of structural linguistics. Nevertheless, there seems to me that there are four more fundamental reasons why the linguistic approach seems to fail in providing the scholar with a satisfactory account of meaning in cinema.

The first reason is that the linguistic view of meaning is simply too narrow and too reductionist. As Mark Johnson aptly puts it, "if you assume that meaning is essentially linguistic and tied to concepts and propositions, then anything in art that is not expressible propositionally is ignored or dismissed as meaningless or cognitively insignificant." ${ }^{69}$ Noël Carroll echoes this claim when he charges that "it is an error to attempt to model all art interpretation on linguistic models."70 He coins this charge the "linguistic fallacy," as an antidote to Wimsatt's and Beardsley's most famous slogan of "the intentional fallacy" (i.e., the disregard of author intentions in the interpretation of art). ${ }^{71}$ It is a fallacy, he argues, "because most artforms-and, therefore, most of the artworks produced in those artforms-are not governed by the kind of structures we find in language proper. That is, the objects of interpretation with respect to most artforms are not language-like in the sense of possessing the kind of rules that 
determine things such as word meaning and sentence meaning."72 Take for example, the pictorial quality of films. Pictures on the whole do not require decoding in order for them to be understood. Comprehension of images is based upon a shared capacity for embodied perception. We grasp the meaning using the same perceptual abilities that enable us to perceive faces and expression in ordinary experience. Consequently, if one defines meaning in film predominantly at the level of the combination of images, as film semiotics does, one misses an important source of meaning.

Secondly, film semiotics may not go as far as to claim that film is a natural language, but by analogizing meaning in visual representations to linguistic signification (and by that, imposing conformity), it unavoidably fails to value the differences between film and language. In other words, the problem is not that film semiotics strive to find a deeper structure that is shared by both film and language-in fact, we will see later that the embodied approach to meaning does just the same-but that the structure they propose (the disembodied, linguistic one) is a very limited one because it diverts attention "from those aspects that may be unique to film."73 In case of film, this implies, above all, that the common structure should be in accordance with, to quote Prince, "a recognition of cinematic images as iconic rather than as symbolic signs, depending on relations of similarity to, rather than difference from, what they represent." ${ }^{\prime 4}$ It is precisely for this reason that the project of film semiotics eventually fails to account for the paradox of cinematic meaning.

Thirdly, the linguistic turn in film studies put an end to a tradition of film theory where the focus has been on a psychological and scientific explanation of films. This tradition goes back as far as the first achievements in psychological research of the film since its earliest beginnings in the 1910s and includes, among others, the writings of Hugo Münsterberg, Rudolf Arnheim and Albert Michotte. ${ }^{75}$ These scholars set themselves the task of describing the psychological mechanisms that explain the perception and comprehension of film. The structuralist or linguistic turn in the 1960s, however, severely undermined the significance of their works by displacing the locus of meaning from the mind to the linguistic sign. This lack of interest in psychology continued unabated in the 1970s and 1980s as film theory began to establish a strong affinity toward interpretative and ideologically driven doctrines. Bordwell calls this development "SLAB theory" as it is mostly based on Saussurean linguistics, Lacanian psychoanalysis, Althusserian Marxism, and Barthian textual theory. ${ }^{76}$ It is also more commonly known as "Grand Theory" because of its all-encompassing and totalizing claims. ${ }^{77}$ In this tradition theorizing does no longer fulfil the function of explaining. Rather it becomes, to quote Carroll, "the routine application of some larger, unified theory to questions of cinema, which procedure churns out roughly the same answers, or remarkably similar answers." 78 It was not until the 1980s and the 1990s, when the "cognitive turn" took its grip in various fields of knowledge, that a handful of scholars, including, among others, Noël Carroll, David Bordwell, Edward Branigan, Ed Tan, Carl Plantinga, Torben Grodal and Murray Smith, started to restore the interdisciplinary link between film studies and the cognitive sciences. This discipline, known today as Cognitive Film Theory, sets itself apart from Grand Theory in its commitment to "clarity of exposition and argument and to the relevance of empirical evidence and the standards of science (where appropriate)." ${ }^{\prime 9}$ Especially in the light of the ever-growing influence of evolutionary theory and recent advances in neuroscience, and the increasing prevalence of digital technologies, such a bridging of the divide between the humanities and the natural sciences, what Smiths recently referred to as the building of a "third cultural" or "naturalized" approach to film and art, is more relevant than ever. ${ }^{80}$ 
Fourthly and perhaps most importantly is that the propositional view of meaning in recent years has lost a great deal of its theoretical weight and rhetorical power as a new paradigm entered the field that took over the place from its intellectual ally, first-generation cognitive science. This shift has been referred to as "second-generation cognitive science" or "the science of the embodied mind." ${ }^{81}$ It is precisely through this new paradigm that the paradox of cinematic meaning will come to the surface again as the mind, and not the sign, reclaims its rightful place as the locus of meaning. ${ }^{82}$

\section{Toward an embodied view of meaning in cinema}

In contrast to first-generation cognitive science, embodied cognitive science treats the mind, concepts, meaning, and rationality as fundamentally embodied, and therefore as not reducible merely to the functional relations and programs of a disembodied machine. Theoretical support for this view is highly disparate and can be derived from various intellectual sources as diverse as linguistics, psychology, anthropology, philosophy and neurophysiology. ${ }^{83}$ Despite a great deal of interpretational variety among these disciplines, they nevertheless share, in lesser or greater degree, the thesis that conceptual structure arises from bodily, social and cultural experience, so part of what makes conceptual organization meaningful are the experiences with which it is associated. Two scholars that have contributed considerably to the theoretical and methodological development of the embodied cognition thesis are the cognitivist linguist George Lakoff and the philosopher Mark Johnson. ${ }^{84}$ Together they proposed a theory of embodied cognition whose central constituent elements might be isolated in the following condensed form:

(1) Conceptual structures arise from the nature of human bodily interaction with the external world.

(2) This connection is captured, among others, by the theoretical notion of an image schema. Image schemas are relatively abstract conceptual patterns that arise directly from our everyday interaction with and observation of the world around us. These patterns are intrinsically meaningful by virtue of their connection to our bodies and our embodied experience. They cannot be characterized adequately by meaningless symbols.

(3) These image schemas, in turn, provide the bodily basis for conceptual metaphors. We recruit their concrete inferential logic in order to reason about abstract target domains. These mappings across domains are captured by the form of "a is b."

(4) In addition, the target domains of conceptual metaphors may also be structured by means of metonymy. In contrast to metaphor, metonymy only involves one conceptual domain. These mappings within a single domain are captured by the form of "a stands for b." 85

A thorough clarification of these elements will be reserved for the second chapter of this book. For now, let us consider the general implications of an embodied account of the mind for our conception of meaning in cinema. 
First, the embodied cognition thesis leads to a fundamentally new hypothesis on the nature of meaning in cinema (and for that matter, the arts in general). ${ }^{86}$ If meaning in film is a matter of conceptual structure, and it is assumed by many that this conceptual structure is not disembodied, but embodied, then it follows that meaning in film is also embodied, that is, "it arises through embodied organism-environmental interactions in which significant patterns are marked within the flow of experience." ${ }^{87}$ This has an important consequence: it implies that meaning in film goes far beyond the confines of words and sentences. That is, in order to study meaning in film, one has to look beyond arbitrary semantics and syntax, and instead focus on the ways that the artful resources of filmmaking can be related to significant patterns of bodily experience.

Second, with the formulation of this new hypothesis comes also the need to readdress the central question inherent to the paradox of cinematic meaning, namely, how does film connect to conceptual structure? Film semioticians evaded this question by displacing the locus of meaning from the mind to the linguistic sign. However, since meaning is assumed to be no longer disembodied, the privileged role of language as the centre of meaning becomes problematic, thus prompting the need to go back to the drawing table to re-establish the connection between mind and film anew, this time from the perspective of the MIND IS BODY metaphor, rather than the THOUght AS LANGUAGe metaphor. ${ }^{88}$ Diagrammatically, this can be represented as in figure I.4.

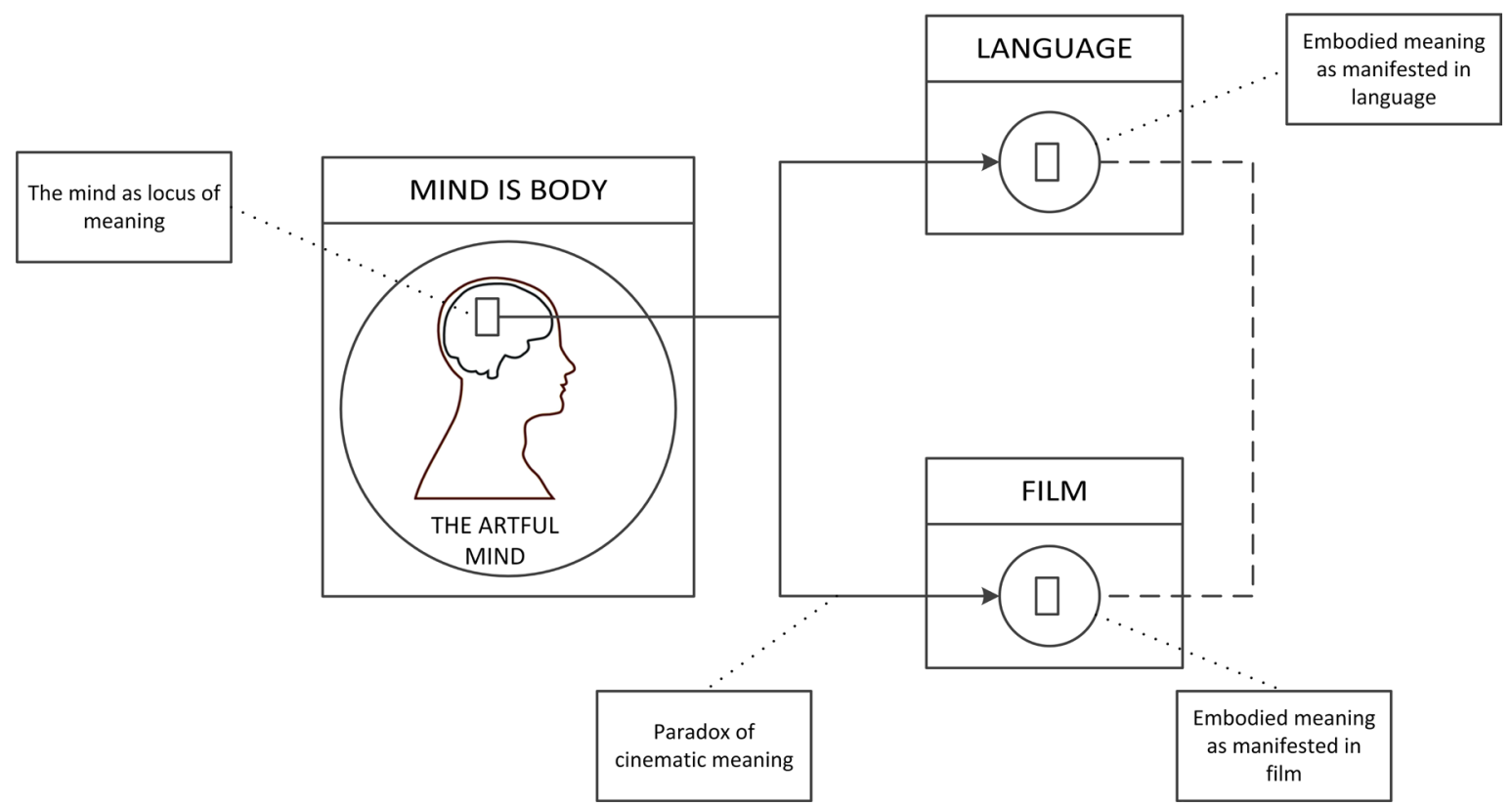

Figure I.4 Readdressing the paradox: The embodied view of meaning in film.

As this figure shows, the harmony between film and language is no longer based on the disembodied linguistic sign, as in figure I.3, but on the notion of embodiment. In this sense it can be said that meaning exceeds linguistic 
meaning (with the latter being just one part of the former). Language, like film or any other art form for that part, is merely one form of expression in which the embodied meaning manifests itself. Metz said precisely the same thing in relation to filmic codes with the crucial difference that the meaning in his model is disembodied. This is the actual weakness of Metz's work rather than the comparison between film and verbal language.

As a consequence of this, the scholar finds himself faced again with the question as to how meaning (that is, embodied meaning this time) can be expressed in an overall iconic medium such as film. The same question with respect to the symbolic medium of language has been rigorously addressed in the field of cognitive linguistics, where a number of abstract concepts have been subjected to an embodied inquiry. Not coincidentally, these concepts overlap to a large extent with the kind of concepts that define the conceptual structure of mental causation, which in terms of narratives is, as we already mentioned above, a rich level of meaning. These concepts include both event-structure concepts (e.g., events, cause, state) as well as concepts denoting mental states (e.g., emotion, perception, thinking). ${ }^{89}$

Although an increase of interest in embodied metaphor can be discerned in the field of (cognitive) film studies, the same kind of academic scrutiny is still missing. ${ }^{90}$ Given Lakoff and Johnson's embodied view of meaning, any systemic account of the question above should at least take into consideration three fundamental tasks. First, to identify the conceptual structure that can be taken as representative of a film's or group of films' intended (situational) meanings. Second, to examine how this structure reflects embodied experience by showing how image-schematic metaphors and metonymies flesh out its literal skeleton. Third, to examine how film reflects this embodied conceptual structure by showing how film may give expression to these image-schematic metaphors and metonymies by its own medium specific tools (e.g., film style, acting, etc.). Baring these three tasks in mind, we can now start to sketch out the structure of this book.

\section{The structure of the book}

The first chapter takes up the challenge of identifying some of the central concepts that define the meanings of Kubrick's films. Given that we have already determined that the focus of this book will be on the situational and referential meanings and given that these meanings are closely tied to characters and their behaviors, we will locate these concepts within the narrative level of Kubrick's films. We first offer a general, conceptual discussion of the notion of narrative, after which we address the question as to where to locate the bare plot descriptions of Kubrick's films. Knowing where to find these conceptual descriptions, we will be able to provide the reader with thirteen scene descriptions, one for each feature film that Kubrick directed. From these descriptions, we will then derive a conceptual skeletal blueprint, a literal meaning-structure of Kubrick's work, that will be argued to underlie all of the thirteen narratives. As already suggested above, this structure will be referred to as the structure of "mental causation."

This structure, in turn, will provide us with all of the central concepts necessary to move toward the second chapter of this book, which aims to address the question of their embodiment. By virtue of which metaphors and 
metonymies are the concepts inherent to the structure of mental causation fleshed out by bodily knowledge? Given that image schemas fulfil a fundamental role in describing the answer to this question, a considerable degree of attention will be devoted to a clarification of this concept. A concise survey of the cognitive linguistic literature will eventually lead us to distinguish between two core metaphor systems of human thinking that account for an understanding of mental causation, namely the EVENT-STRUCTURE metaphor and the MIND IS A BODY metaphor. ${ }^{92}$

Together, the first two chapters constitute the "conceptual" part of this book. The next part, by contrast, considers the task of showing how the embodied conceptual structure, as identified in the first chapter, is fleshed out visually in the films of Kubrick. Likewise, this "formal" part will be divided over two chapters.

The third chapter is general and considers the question as to how the constituents of embodied meaning-making, as discussed in chapter 2, can be manifested in film. For practical reasons, and given that the visual level is the most essential to our understanding of cinema, we will narrow down our focus to the iconic surface level of moving pictures. Given the two-fold nature of conceptual metaphor, this task will amount to assessing two key questions: (1) How may image schemas be imposed onto the iconic and representational level of films? and (2) How may moving pictures give rise to target domains to which these image schemas might be extended metaphorically? Answers to these questions will be sought in the notions of film style and acting, respectively.

It is only once we have successfully dealt with both challenges that we will be able to move to the fourth chapter in which we shall consolidate all of the insights of the previous chapters by exploring the question as to how the films of Kubrick resort to image schemas for the purpose of conveying the stories of mental causation visually. It is in the course of closely analysing various film scenes that we will gradually come to address the paradox of cinematic meaning, as raised at the beginning of this introduction.

Lastly, we take the opportunity of the concluding chapter to assess two theoretical dilemmas that involve the film viewer. The first dilemma takes into consideration the question as to how viewers are able to discover the situational meanings of Kubrick's films given the fact that they, as observers, are not performing the bodily actions that lie at the heart of the visual conveyance of those meanings. In addressing this question, we will stress the importance of embodied simulation mechanisms inside the viewer as they have received considerable attention among various scholars working on the boundary between film art and cognitive science. ${ }^{93}$ The second dilemma takes into account the paradoxical relationship between meaning and (film) music as already mentioned above. How can non-representational, instrumental music, as prominently present in Kubrick's films, be experienced as meaningful (e.g., as expressing the emotions of characters), given the fact that musical sounds, unlike words or visual images, do not refer, strictly speaking, to something outside themselves? Following an embodied cognitive approach to musical meaning, we will locate the answer to this question not so much in the music itself, but in the embodied viewer who makes sense of the music. Music may not possess the bodily and spatial properties that are necessary for fleshing out the abstract meaning (e.g., motion, gravity, containment), the people who listen to it nonetheless resort to these properties in order to understand and conceptualize the musical sounds they hear. It is precisely in this sense that we will argue that music can be capable of expressing meaning including the concepts of mental causation. Viewers are able to do so because the embodied tools we use to conceptualize music are similar to the embodied tools we use to understand the meaning. 


\section{Notes}

1. Ciment, Kubrick, 187. This quote is Jeremy Barham's own translation from the original French edition published by Calmann-Lévy. See Barham, "Incorporating Monsters," 141. Strangely enough, these words are not included in Faber and Faber's English translation.

2. The major events in Kubrick's personal life are well documented, so we will not elaborate on them here. Readers interested in Kubrick's biography may find the following readings of interest: Baxter, Stanley Kubrick; LoBrutto, Kubrick; and Abrams, Stanley Kubrick. See in this regard also the documentary made by his long-time assistant and brother-in-law Jan Harlan entitled Stanley Kubrick: A Life in Pictures (2001).

3. This can be observed in the flow of publications that continues unabated. Some recent collective works on Kubrick and his films include, among others, Broderick, The Kubrick Legacy; Fenwick, Understanding Kubrick's 2001; Fenwick, Hunter and Pezzotta, "Stanley Kubrick"; Hunter, "Kubrick and Adaptation”; Ljujić, Krämer and Daniels, Stanley Kubrick; and Szaniawski, After Kubrick.

4. Benson, Space Odyssey, 8.

5. LoBrutto, Stanley Kubrick, 277. For a good compilation of interviews taken between 1959-1987, see Philips, Stanley Kubrick.

6. Rapf, "A Talk With Stanley Kubrick," 78. Very enlightening in this regard is also Kubrick's own one-page article entitled "Words and Movies," 14. For similar quotations in which the filmmaker criticizes the linguistic view of meaning in cinema, see, among others, Nordern, "Playboy Interview," 47-48; and Belson, Space Odyssey, 365.

7. Here we are paraphrasing Kubrick who in an interview with Joseph Gelmis stated that "movies present the opportunity to convey complex concepts and abstractions without the traditional reliance on words." See Gelmis, "The Film Director as Superstar," 90.

8. Later in this book, we will challenge this premise, but for now let us take it as given.

9. Grice, “Meaning," 383.

10. Searle, "Grice on Meaning," 11.

11. Carroll, "Art Interpretation," 119-120. This is very close in spirit to what Paisley Livingston refers to as "the meshing condition", "the intention to mean $q$ by saying or otherwise representing $p$ is successful just in case the intention to imply $q$ meshes sufficiently with what is written, spoken, or otherwise put on display." See Livingston, Cinema, Philosophy, Bergman, 99.

12. It is important to keep in mind that although the embodiment of $\mathrm{y}$ in $\mathrm{x}$ is a necessary condition for the audience's recognition of $\mathrm{y}$, it is not a sufficient condition. The fact that $\mathrm{y}$ is embodied in $\mathrm{x}$ does not in itself guarantee the audience's discovery of $\mathrm{y}$.

13. See, for instance, Davidson, "Truth and Meaning"; and Searle, Speech Acts.

14. For a good recent discussion of truth-based approaches to linguistic meaning, see Iten, Linguistic Meaning.

15. Evans and Green, Cognitive Linguistics, 156. Among the foundational book-length texts in cognitive semantics are, Talmy, Cognitive Semantics; Langacker, Cognitive Grammar; Lakoff and Johnson, Metaphors; Lakoff, Women; and Johnson, The Body in the Mind.

16. Lakoff and Johnson, Philosophy; Johnson, Embodied Mind; Gibbs, Embodiment.

17. Wilson and Sperber, Meaning and Relevance, 2.

18. Evans and Green, Cognitive Linguistics, 7.

19. Reddy, "The Conduit Metaphor," 167.

20. In chapter 2 of this book, when clarifying the notion of embodied cognition, the concept of "container" will be identified as a prototypical example of what Lakoff and Johnson term an "image schema," that is, a dynamic and recurrent pattern of sensory-motor experience. From this perspective, ENTRY and exit designate two "dynamic patterns of containment." See also Dewell, "Dynamic patterns," 369-394.

21. For a discussion of cinematic authorship, see Livingston, "Cinematic Authorship," 132-148; and Gaut, "Film Authorship," 149-172. See also Meskin, "Authorship," 12-28; and Sellors, "Collective Authorship," 263-271.

22. Sellors, "Collective Authorship," 263.

23. Turner, The Artful Mind.

24. Philips, Stanley Kubrick, vii.

25. Young, "The Hollywood War," 7. 
26. Livingston, "Cinematic Authorship," 135; Sellors, "Collective Authorship," 266. Sellors prefers "filmic author" to "cinematic author" because the former evokes the medium of film, whereas the latter suggests the institution of cinema. He defines a filmic author as "the agent or agents who intentionally token(s) a filmic utterance, where 'to token' refers to any action, an intended function of which is to make manifest or communicate some attitude(s) by means of the production of an apparently moving image projected on a screen or other surface and a filmic utterance is the result of the act of tokening in this medium."

27. Bordwell, Making Meaning, 8-9.

28. Ibid., 2.

29. Van Dijk and Kintsch, Strategies in Discourse Comprehension.

30. Persson, Understanding Cinema, 31.

31. Kim, Philosophy of Mind, 173-204; Robb and Heil, "Mental Causation."

32. Bordwell, Making Meaning, 2.

33. Persson, Understanding Cinema, 34. The interpretation of the monolith as screen can be credited to Loughlin, Alien Sex, 73. For a good discussion of the seemingly endless cycle of interpretations in The Shining, see Lovisato, "(Do Not) Overlook."

34. Ibid., 34 .

35. Bordwell and Thompson, Film Art, 56.

36. Ibid., 56 .

37. "Tectonics of the Mechanical Man" is a chapter in the book A Cinema of Loneliness, Kolker's classic account of the New Hollywood. See also Kolker, Extraordinary Image.

38. Over the last years many Kubrick scholars have adopted a more overt formal approach to Kubrick's films. They include, among others, Coëgnarts, Stanley Kubrick; Falsetto, Stanley Kubrick; Kuberski, Kubrick's Total Cinema; Luckhurst, The Shining; McQuiston, We'll Meet Again; and Sperl, Die Semantisierung.

39. Wilson, "Interpretation," 163.

40. Young, "The Hollywood War," 7.

41. See, for instance, Prince, "The Discourse of Pictures," 16-28.

42. Chandler, Semiotics, 36.

43. Eco, "Iconic Signs," 1.

44. Gaut, Cinematic Art, 52.

45. Scruton, The Aesthetics of Music, 118.

46. Walton, "What Is Abstract," 351.

47. For notable references to language in early film theory, see, among others, Eisenstein, The Film Sense; Lindsay, The Art of the Moving Picture; Pudovkin, Film Technique, xiii-xvii; and Spottiswoode, A Grammar of the Film. Important studies within the field of film semiotics include, among others, Bettetini, The Language and Technique of the Film; Carroll, Structural Psychology; Eco, "Cinematic Code"; Metz, Film Language, Language and Cinema; and Peters, Pictorial Signs. For good reflective discussions of the relationship between film and language, see, among others, Buckland, "Film Semiotics"; Prince, “The Discourse of Pictures"; Pryluck, "The Film Metaphor"; and Stam, "Film and Language."

48. See also Carroll, Structural Psychology, 29.

49. We shall see in chapter 5 of this book that the same observation can also be made with regard to music. Johnson calls this the MUsic As LANGUAGE metaphor. For a discussion, see Johnson, The Meaning of the Body, 235.

50. Carroll, Structural Psychology, 31; see also Buckland, "Film Semiotics," 88.

51. Ibid., 1.

52. See also Buckland, "Film Semiotics," 88.

53. Metz, Language and Cinema, 150.

54. Lakoff and Johnson, Philosophy, 75. For a discussion, see also Johnson, Embodied Mind, 16. 
55. Chomsky, Syntactic Structures.

56. Johnson, Embodied Mind, 16.

57. Ibid., 69. For a discussion of this hypothesis, see Fodor, The Language of Thought.

58. Barsalou, "Perceptual Symbol Systems," 578-579.

59. Johnson, The Meaning of the Body, 202.

60. Ibid., 202.

61. Lakoff and Johnson, Philosophy in the Flesh, 244.

62. Iten, Linguistic Meaning, 1-6.

63. Johnson, The Meaning of the Body, 272.

64. Buckland, The Cognitive Semiotics of Film, 18

65. Ibid., 2. Film writings that draw on insights from first-generation cognitive science, include, among others, Chateau, "Generative Model of Film Discourse”; and Colin, "The Grande Syntagmatique Revisited.”

66. Carroll, The Philosophy of Motion Pictures; Currie, Image and Mind; Prince, "Psychoanalytical Film Theory"; and Pryluck, "The Film Metaphor." For a short summary of Carroll's and Currie's critiques, see Sinnerbrink, New Philosophies of Film, 24-27.

67. See, for instance, Currie, Image and Mind.

68. Buckland, "Film Semiotics", 99.

69. Johnson, "Identity, Bodily Meaning, and Art" 21.

70. Carroll, “Art Interpretation,” 117.

71. Wimsatt and Beardsley, "The Intentional Fallacy."

72. Carroll, “Art Interpretation," 122.

73. Pryluck, "The Film Metaphor," 123.

74. Prince, "Psychoanalytical Film Theory," 80.

75. For an excellent overview of their achievements, see Tan, "A Psychology of the Film."

76. Bordwell, "Historical Poetics," 385.

77. Bordwell, "Contemporary Film Studies"; Carroll, "Prospects for Film Theory."

78. Ibid., 41

79. Plantinga, "Cognitive Film Theory," 258. For a good overview of some of the current views and issues within cognitive film theory, see Shimamura, Psychocinematics; and Nannicelli and Taberham, Cognitive Media Theory.

80. Smith, Film, Art, and the Third Culture.

81. Lakoff and Johnson, Philosophy in the Flesh, 77. For a discussion, see also Johnson, Embodied Mind, 17.

82. The use of the word "new" here is not entirely accurate as the embodied view of the mind is not a completely new paradigm, but rather a continuation, as Barsalou has pointed out, of a tradition of thinking that was considered dominant until the early twentieth century. Its historical roots go back as far as Ancient Greek Philosophy (Aristotle and Epicurus) and includes, among others, the intellectual heir of British empiricism (Locke, Berkeley, and Hume), American pragmatism (John Dewey) and French phenomenology (Merleau-Ponty, Bergson). See Barsalou, “Grounded Cognition,” 619.

83. For a good discussion of some of its central claims, see Wilson, "Embodied Cognition."

84. Seminal works include Lakoff and Johnson, Metaphors; Lakoff and Johnson, Philosophy; Lakoff, Women; Johnson, The Body in the Mind; and Johnson, The Meaning of the Body.

85. Although metonymy has been less extensively investigated by Lakoff and Johnson, it constitutes a fundamental aspect of recent cognitive linguistic literature. See, among others, Evans and Green, Cognitive Linguistics. Hence, the reason why we include it in this overview.

86. In contrast to Lakoff, Johnson himself has written a great deal about the relationship between the arts and embodied meaning. Worth reading in this context are Johnson, The Meaning of the Body, "Identity, Bodily Meaning, and Art", and more recently, The Aesthetics of Meaning and Thought. 
87. Johnson, The Meaning of the Body, 273

88. Lakoff and Johnson, Philosophy, 235.

89. For a discussion of event-structure concepts, see Lakoff and Johnson, Philosophy, 170-234. For a pioneering work on the metaphorical conceptualization of emotion, see Kövecses, Metaphor and Emotion. Among the influential works on the mental concepts of perception and thinking are Lakoff, "Reflections"; Yamanashi, "Metaphorical Modes"; Lakoff and Johnson, Philosophy, 393-399; Yu, "Chinese Metaphors", "The Eyes"; Gibbs, Embodiment, 97; and Johnson, The Meaning of the Body, 165. We will discuss these and other more in detail in chapter 2 of this book.

90. Studies that apply insights from Conceptual Metaphor Theory to film include, among others, Buckland, The Cognitive Semiotics of Film; Branigan, Projecting a Camera; Kappelhoff and Müller, "Embodied Meaning Construction"; Fahlenbrach, "Emotions in Sound"; Ortiz, "Primary Metaphors"; Coëgnarts and Kravanja, "Embodied Visual Meaning”; Winter, "Horror Movies." Two edited volumes at the crossroads of CTM and film studies, are Coëgnarts and Kravanja, Embodied Cognition and Cinema; and Fahlenbrach, Embodied Metaphors in Film, Television, and Video Games.

91. For a discussion of the EVEnT-STruCture metaphor, see Lakoff and Johnson, Philosophy, 178-194. For a discussion of the Mind is A BODY metaphor, see Lakoff and Johnson, Philosophy, 235-236; and Sweetser, From Etymology to Pragmatics.

92. See, among others, Hasson et al., "Neurocinematics"; Grodal, Embodied Visions; Gallese and Guerra, "Embodying Movies"; D’Aloia and Eugeni, "Neurofilmology"; Coëgnarts, "Cinema and the Embodied Mind." 
